\title{
Salvage chemotherapy with procarbazine and fotemustine combination in the treatment of temozolomide treated recurrent glioblastoma patients
}

\author{
Antonio Silvani - Elena Lamperti - Paola Gaviani - Marica Eoli · \\ Anna Fiumani - Andrea Salmaggi - Chiara Falcone - Graziella Filippini · \\ Andrea Botturi · Amerigo Boiardi
}

Published online: 8 February 2008

(C) Springer Science+Business Media, LLC. 2008

\section{Erratum to: J Neurooncol}

DOI 10.1007/s11060-007-9427-y

There were errors in Table 2 of the original publication. The corrected values are shown in bold in Table 2 below. The corresponding values in the text of the original publication were correct (2nd and 3rd paragraph of left column on the same page).

Table 2 Overall Median PFS and 6 months-PFS from the start of PCB-FTM

ne $=100 \%$ of progression or censored before 6 months

\begin{tabular}{lll}
\hline & Median PFS: weeks (95\% CI) & PFS-6: \% (95\% CI) \\
\hline Whole group of 54 recurrent glioblastoma patients & \\
$19.3(13.7-24.4)$ & $26.7(10.6-42.8)$ \\
II line chemotherapy: 31 patients treated at first recurrence-progression & \\
$19.7(13.7-25.2)$ & $\mathbf{1 7 . 1}(\mathbf{0 - 3 4 . 3})$ \\
Reoperated & $19.7(13.6-26.1)$ & ne \\
Non-reoperated & $16.7(6.8-26.5)$ & $42.8(8.8-77)$ \\
III line chemotherapy: 23 patients treated at second recurrence-progression & \\
& $19.3(\mathbf{6 . 8}-32.1)$ & $\mathbf{4 5 . 8}(\mathbf{1 6 . 6}-\mathbf{7 5})$ \\
Reoperated & $25.7(13.3-38.6)$ & $50.9(19.1-82.7)$ \\
Non-reoperated & ne & ne \\
\hline
\end{tabular}

The online version of the original article can be found under doi:10.1007/s11060-007-9427-y.

\footnotetext{
A. Silvani $(\bowtie) \cdot$ E. Lamperti · P. Gaviani · M. Eoli .

A. Fiumani · A. Salmaggi - A. Botturi · A. Boiardi Department of Neuro-oncology, Fondazione IRCCS Istituto Neurologico "Carlo Besta", Via Celoria, 11, Milan 20133, Italy

e-mail: silvani@istituto-besta.it

C. Falcone · G. Filippini

Neuro-epidemiology Unit, Fondazione IRCCS Istituto

Neurologico "Carlo Besta", Via Celoria, 11, Milan 20133, Italy
} 